\title{
Advanced MRI Morphologic Study Shows No Atrophy in Healthy Individuals with Hippocampal Hyperintensity
}

\author{
A. Labate, A. Cerasa, A. Cherubini, U. Aguglia, A. Quattrone, and A. Gambardella
}

\begin{abstract}
SUMMARY: We have already shown that brain MR imaging of healthy individuals frequently reveals either unilateral or bilateral Hh, which is considered a hallmark of hippocampal sclerosis. We performed a follow-up (5-year interval) clinical and advanced imaging study of these individuals to address whether Hh may have masked occult brain atrophy or contributed to a later onset of epilepsy. Subjects with Hh ( $n=$ 13) underwent a detailed clinical-imaging protocol, with a 3T scan and were studied with automated hippocampal segmentation (FreeSurfer), whole brain voxel-based morphometry, and shape analysis. All 13 subjects with $\mathrm{Hh}$ had normal neurologic examination findings with no cognitive impairment. Multimodal structural neuroimaging methods did not show clear evidence of significant volumetric changes between subjects with or without $\mathrm{Hh}$. We clearly showed that $\mathrm{Hh}$ is not associated with any occult brain atrophy; furthermore, none of the healthy subjects with MR imaging evidence of Hh developed epilepsy or trouble with cognition.
\end{abstract}

ABBREVIATIONS: $\mathrm{FDR}=$ false discovery rate; $\mathrm{Hh}=$ hippocampal hyperintensity; $\mathrm{Hs}=$ hippocampal sclerosis; $\mathrm{TLE}=$ temporal lobe epilepsy; VBM = voxel-based morphometry

S ince the 1990s, MR imaging evidence of hippocampal atrophy and Hh has become a highly sensitive and specific marker of Hs and TLE. ${ }^{1-3}$ More recently, we have shown that MR imaging evidence of $\mathrm{Hs}$ is present in a third of patients with drug-responsive TLE, a finding that indicates that $\mathrm{Hs}$ itself does not necessarily mean intractable epilepsy. ${ }^{3}$

In daily clinical practice, it has become increasingly clear that MR imaging signs of Hs must always be carefully interpreted because they may occur as incidental findings in patients without epilepsy. ${ }^{4}$ Most important, we recently found, in approximately $25 \%$ of healthy controls, either unilateral or bilateral $\mathrm{Hh}$, whereas hippocampal atrophy, whether or not associated with hyperintensity, was seen exclusively in patients with TLE. ${ }^{5}$ Obviously, we could have potentially missed minor hippocampal anomalies contributing to $\mathrm{Hh}$, and we could not definitively exclude a later onset of epilepsy.

In the present study, we sought to address these issues by conducting a 3T MR imaging study in the same population of controls. We also used 3 different advanced structural neuroimaging

Received October 23, 2012; accepted after revision November 14.

From the Institute of Neurology (A.L., U.A., A.Q., A.G.), University Magna Græcia, Catanzaro, Italy; Neuroimaging Research Unit (A.L., A.C., A.C., A.Q.), Institute of Neurological Sciences, National Research Council, Germaneto, Italy; and Institute of Neurological Science (A.G.), National Research Council, Cosenza, Italy.

Please address correspondence to Angelo Labate, MD, Clinica Neurologica, Università degli Studi “Magna Græcia,” Campus Universitario Germaneto, Viale Europa, 88100 Catanzaro, Italy; e-mail: labate@unicz.it

http://dx.doi.org/10.3174/ajnr.A3458 operator-independent approaches, called shape analysis, ${ }^{6}$ voxelbased morphometry, ${ }^{7,8}$ and automated hippocampal segmentation (FreeSurfer; http://surfer.nmr.mgh.harvard.edu/) ${ }^{9,10}$ to determine whether $\mathrm{Hh}$ seen on visual MR imaging could have masked occult atrophy.

\section{METHODS}

\section{Subjects and MR Imaging Protocol}

Since January 2012, fifty-one healthy volunteers were recontacted from our previous study. Four subjects were excluded due to unavailability. The remaining 47 controls (24 women; mean age, $39.3 \pm 10.8$ years) underwent telephone interviews by 2 trained epileptologists (A.L. and A.G.) to document the absence of seizures and normal neurologic history in each individual. None of the volunteers had any contraindications to MR imaging, and they were re-invited to undergo MR imaging as part of our neuroimaging study. Brain MR imaging was performed according to our routine protocol ${ }^{11}$ by a $3 \mathrm{~T}$ scanner with an 8 -channel head coil (Discovery MR750; GE Healthcare, Milwaukee, Wisconsin) at the Neuroimaging Research Unit, Institute of Neurologic Sciences, National Research Council, Catanzaro, Italy.

Structural MR imaging data were acquired by using a 3D T1weighted spoiled gradient-echo sequence with the following parameters: TR, $3.7 \mathrm{~ms}$; TE, $9.2 \mathrm{~ms}$; flip angle, $12^{\circ}$; voxel-size, $1 \times$ $1 \times 1 \mathrm{~mm}^{3}$. Subjects were positioned comfortably in the scanner with a forehead-restraining strap and various foam pads to ensure head fixation. The MR imaging protocol also included (axial and 
coronal sections) T2-weighted images (TR, $4613 \mathrm{~ms}$; TE, $102 \mathrm{~ms}$; image matrix, $512 \times 512$; FOV, $24 \mathrm{~cm}$; 36 sections; $4.0-\mathrm{mm}$ sections; 0-mm gap); axial fast FLAIR images (TR, $9500 \mathrm{~ms}$; TE, 100 ms; image matrix, $512 \times 256$; FOV, 24 cm; 32 sections; $3.5-\mathrm{mm}$ sections, 0-mm gap); and sagittal 3D TSE with variable flip angle FLAIR images (TR, 8000 ms; TE, 134 ms; TI, 2200; image matrix, $256 \times 256$; FOV, $25.6 \mathrm{~cm}$; 148 sections; $1.2-\mathrm{mm}$ sections; $0-\mathrm{mm}$ gap). The MR imaging diagnosis of $\mathrm{Hh}$ was based on the occurrence of the neuroimaging alterations that are considered reliable indicators of Hh: an increased mesial temporal signal intensity alteration on FLAIR or T2 images, or both. ${ }^{1,3}$

Of the entire group, 13 subjects showed FLAIR Hh at previous scanning, confirmed by $3 \mathrm{~T}$; these subjects with Hh were compared with a matched group without $H$ h. The mean follow-up was 5 years (range, 4-6 years). They also underwent neurologic and cognitive evaluations. The participants signed a consent form approved by the University Committee for Protection of Human Subjects in Research.

\section{Shape Analysis}

T1-weighted images were processed by using the fMRI of the Brain Software Library 4.1 (http://fsl.fmrib.ox.ac.uk/fsl/fsl-4.1.9/ fsl/linux.html) tools. FMRIB's Integrated Registration Segmentation Tool, Volume 1.2, was used to automatically segment ROIs and to model surface, ${ }^{6}$ and left and right hippocampi were segmented on each subject. The shape differences between groups were evaluated by a multivariate F-test performed on each vertex separately. Only differences surviving correction for multiple comparison (FDR), with a statistical threshold of $P<.05$, were considered. Shape-analysis findings were represented by a $3 \mathrm{D}$ image of the structures in which a colorimetric scale (representing the F-value) highlighted significant differences. Red areas corresponded to regions with no significant shape differences.

\section{VBM Data Processing and Analysis}

Data were processed and examined by using the SPM8 software (http://www.fil.ion.ucl.ac.uk/spm), and we applied VBM implemented in the VBM8 toolbox with default parameters, incorporating the Diffeomorphic Anatomical Registration Through Exponentiated Lie Algebra (SPM5) toolbox, which was used to obtain a high-dimensional normalization protocol. ${ }^{8}$ Images were bias-corrected, tissue-classified, registered, modulated, and smoothed with a Gaussian kernel of 8-mm full width at half maximum.

The gray matter volume maps were statistically analyzed by using the general linear model based on Gaussian random field theory. We investigated the presence of volumetric changes between groups by using analysis of covariance, including age and total intracranial volume as covariates of no interest. Because the Hh group was significantly older with respect to the non-Hh group $(t=-2.95$; $P$ value $<.005)$, to exclude possible spurious effects depending on age, we re-ran our analysis, splitting the group without Hh into 2 cohorts: the younger (including all subjects from 20 to 39 years of age $[n=21]$; mean age, $30.1 \pm 5.2$ years; 12 women) and the older (including subjects from 39 to 60 years [ $n=13]$; mean age, $47.7 \pm 7.4 ; 7$ women) groups.

Statistical analysis was performed within and outside ROIs including the bilateral hippocampi and the parahippocampal gyrus. The statistical threshold was set at $P<.05$, with FDR correction for multiple comparisons within ROIs. We reported other brain regions that were not predicted a priori but met a wholebrain statistical threshold FDR $<.05$.

\section{Automated Hippocampal Volumetry}

To corroborate shape and voxel-based findings, we further performed automated labeling and quantification of hippocampal volume by using FreeSurfer 5.0. The automated procedures for volumetric measures of several deep GM structures have been previously described. ${ }^{9,12}$ This procedure automatically provided segments and labels for up to 40 unique structures and assigned a neuroanatomic label to each voxel in an MR imaging volume based on probabilistic information estimated automatically from a manually labeled training set.

The automated subcortical segmentation performed by FreeSurfer required these steps: First, an optimal linear transform is computed that maximizes the likelihood of the input image, given an atlas constructed from manually labeled images. A nonlinear transform is then initialized with the linear one, and the image is allowed to further deform to better match the atlas. Finally, a Bayesian segmentation procedure is performed, and the maximum a posteriori estimate of the labeling is computed. This approach provides advantages similar to those of manual ROI drawing, ${ }^{13,14}$ without the potential for rater bias, offering an anatomically accurate rendering of regional volumes. ${ }^{10}$ Total intracranial volume was calculated and was used to correct the regional brain volume measurements. ${ }^{15}$ Normalized hippocampal values were calculated as follows: [raw hippocampal volume/total intracranial volume $] \times 1000 .{ }^{10}$

\section{Statistical Analysis}

Categoric variables are expressed as frequency and percentages, and the differences among groups in the hippocampal volumetry were assessed by using an unpaired $t$ test. To evaluate the agreement between 2 readers and the agreement between 2 different measurements, we calculated the Cohen $\kappa$ coefficient and used the Wald statistic to test the null hypothesis that $\kappa$ is equal to zero. Statistical analyses were performed with Statistical Package for Social Science software (Version 15.0; SPSS, Chicago, Illinois) for Windows.

\section{RESULTS}

\section{Clinical, Shape, and VBM Findings}

None of the healthy subjects with or without MR imaging evidence of Hh had developed epilepsy or had a history of febrile convulsions. Neurologic and cognitive examination findings were always normal. None of the subjects had past or current history of vascular diseases, infections, or closed head injury. Furthermore, in the group of subjects with $\mathrm{Hh}$, the MR imaging did not show any brain changes other than $\mathrm{Hh}$.

Comparison of subjects with and without Hh matched for age and sex shape analysis did not detect any structural difference between the 2 groups, either in right or left hippocampal shapes $\left(P_{\mathrm{FDR}}=.0683\right.$; Fig 1$)$. VBM analysis did not demonstrate evidence of significant volumetric changes between the 2 groups 


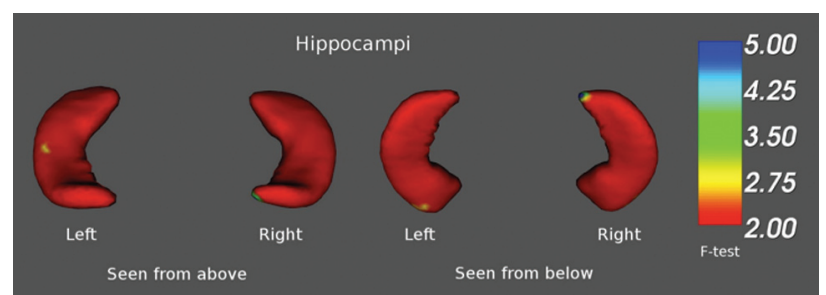

FIG 1. Colorimetric scale of hippocampi expressed with F-values. Red areas correspond to regions with no significant differences.

when either whole-brain or ROI analyses were performed. A slight difference was detected in the head of the left hippocampus when comparing only individuals without Hh according to age (younger and older subjects). These individuals showed reduced gray matter volume with respect to younger age, though this difference did not reach a significant threshold (x: $-15 ; \mathrm{y}: 2 ; \mathrm{z}:-18$, $P_{\mathrm{FDR}}=.483$ ), thus demonstrating that the impact of age on the main effect of group was negligible.

Automated labeling of the hippocampus, as provided by FreeSurfer, confirmed all previous findings, demonstrating only a trend toward loss of gray matter volume in the right $(t$ value $=$ $0.96 ; P$ value $>.35)$ and the left hippocampi $(t$ value $=0.79 ; P$ value $>.46$ ) in individuals with $\mathrm{Hh}$ (Fig 2).

\section{DISCUSSION}

Our results illustrated that Hh was not associated with any localized or widespread cerebral atrophy because both advanced MR imaging methods showed no differences between healthy subjects with or without Hh. Moreover, none of the subjects with MR imaging evidence of Hh have developed epilepsy, any other neurologic diseases, or cognition trouble after 5 years of follow-up. The major implication of these findings is that the incidental MR imaging detection of hippocampal hyperintensities in healthy individuals is common and not significant, so Hh must be prudently judged in the proper clinical context, keeping in mind the high rate of an incorrect diagnosis of epilepsy. ${ }^{16}$

In our previous work, ${ }^{5}$ the 2 possible criticisms were the lack of follow-up in the population studied and the possibility that subtle hippocampal variances could have been missed by visual inspection and could have been the contributors to such MR imaging hyperintensities. For this reason, we have decided to wait for a meaningful follow-up in the same population and to study the whole brain and specific brain regions by using 3 different MR imaging techniques (operator-independent) because of their complementariness. ${ }^{6,8,9} \mathrm{VBM}$ is a morphologic technique that performs a statistical mapping of differences in brain morphology voxel-by-voxel, which produces gray matter "attenuation" or "concentration" measures or volume differences between the 2 groups; alternatively, manual/automatic volumetry is a quantitative measurement of specific brain regions in individual brains. ${ }^{9,10}$ Quantitative morphologic assessment of individual brain structures is often based on volumetric measurements. Volume changes are intuitive features because they might explain atrophy or dilation due to illness. On the other hand, structural changes at specific locations are not sufficiently reflected in volume measurements. For this reason, shape analysis has become of increasing interest to the neuroimaging community due to its potential to precisely locate morphologic changes between healthy and pathologic structures. ${ }^{6}$ The fact that all these advanced neuroimaging approaches depict the same neuroanatomic picture speaks to the robustness of our findings.

\section{CONCLUSIONS}

These results provide the first evidence that Hh frequently observed in healthy volunteers is not potentially associated with occult hippocampal or widespread atrophy that can contribute to such hyperintensities. It is, therefore, probable that such signal hyperintensities may have to do with the hippocampus histologic features because the hippocampus has higher water content, a different cellular composition, and a higher vascular attenuation than that of the other cortices, which may affect signal intensity on brain MR images. ${ }^{5}$ Further advanced neuroimaging studies using different MR imaging parameters, such as T2-weighted images, ${ }^{17}$

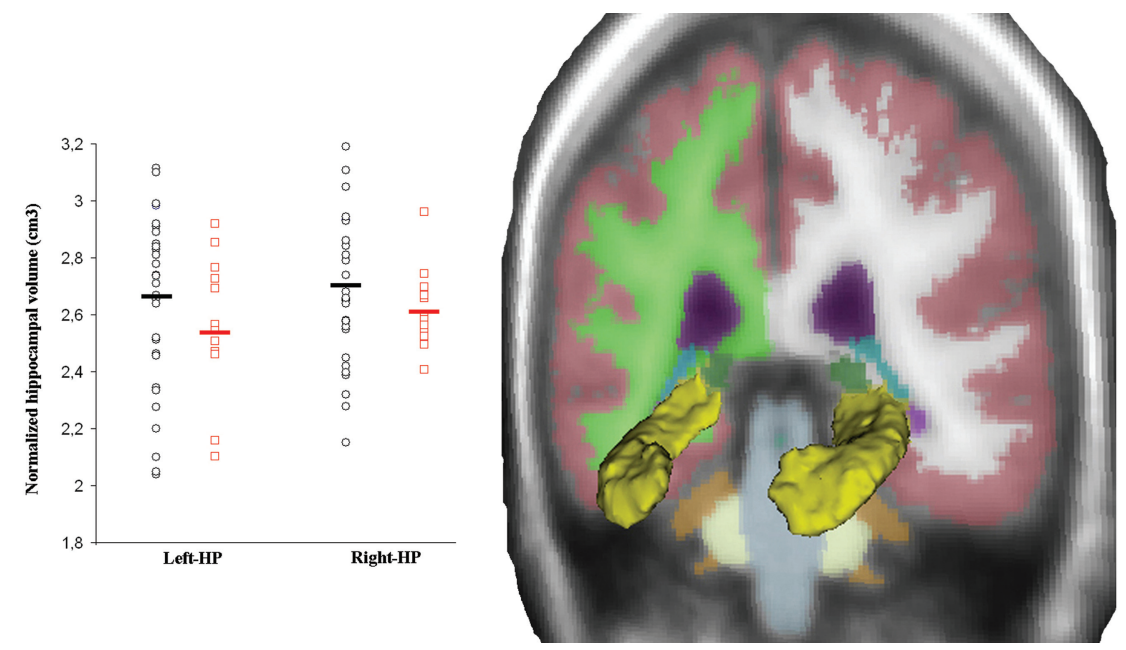

FIG 2. Sample color-coded subcortical segmentation results. 3D surface models, created with 3D Slicer, Version 3 (www.slicer.org), are derived from the FreeSurfer subcortical segmentation of the hippocampus (yellow). No significant volumetric differences were detected between individuals with (red square) and without $\mathrm{Hh}$ (black circle) in the left ( $P$ value $>.46)$ and right hippocampi $(P$ value $>.35)$. Scatter plot of the mean normalized volumes of the left and right hippocampi for each single subject has been plotted on the left side. 
are warranted to better delineate the neurobiologic underpinnings of hippocampal hyperintensities.

\section{REFERENCES}

1. Jackson GD, Berkovic SF, Duncan JS, et al. Optimizing the diagnosis of hippocampal sclerosis using MR imaging. AJNR Am J Neuroradiol 1993;14:758-62

2. Kobayashi E, D’Agostino MD, Lopes-Cendes I, et al. Hippocampal atrophy and T2-weighted signal changes in familial mesial temporal lobe epilepsy. Neurology 2003;60:405-09

3. Labate A, Ventura P, Gambardella A, et al. MRI evidence of mesial temporal sclerosis in sporadic "benign" temporal lobe epilepsy. Neurology 2006;66:562-65

4. Benbadis SR, Tatum WO, Murtagh FR, et al. MRI evidence of mesial temporal sclerosis in patients with psychogenic nonepileptic seizures. Neurology 2000;55:1061-62

5. Labate A, Gambardella A, Aguglia U, et al. Temporal lobe abnormalities on brain MRI in healthy volunteers: a prospective case-control study. Neurology 2010;74:553-57

6. Patenaude B, Smith SM, Kennedy DN, et al. A Bayesian model of shape and appearance for subcortical brain segmentation. Neuroimage 2011;56:907-22

7. Labate A, Cerasa A, Gambardella A, et al. Hippocampal and thalamic atrophy in mild temporal lobe epilepsy: a VBM study. Neurology 2008;71:1094-101

8. Ashburner J. A fast diffeomorphic image registration algorithm. Neuroimage 2007:38:95-113
9. Fischl B, Dale AM. Measuring the thickness of the human cerebral cortex from magnetic resonance images. Proc Natl Acad Sci U S A 2000;97:11050-55

10. Cerasa A, Messina D, Nicoletti G, et al. Cerebellar atrophy in essential tremor using an automated segmentation method. AJNR Am J Neuroradiol 2009;30:1240-43

11. Cerasa A, Passamonti L, Valentino P, et al. Cerebellar-parietal dysfunctions in multiple sclerosis patients with cerebellar signs. Exp Neurol 2012;237:418-26

12. Fischl B, Salat DH, Busa E, et al. Whole brain segmentation: automated labeling of neuroanatomical structures in the human brain. Neuron 2002;33:341-55

13. Morey RA, Petty CM, Xu Y, et al. A comparison of automated segmentation and manual tracing for quantifying hippocampal and amygdala volumes. Neuroimage 2009;45:855-66

14. Pardoe HR, Pell GS, Abbott DF, et al. Hippocampal volume assessment in temporal lobe epilepsy: how good is automated segmentation? Epilepsia 2009;50:2586-92

15. Buckner RL, Head D, Parker J, et al. A unified approach for morphometric and functional data analysis in young, old, and demented adults using automated atlas-based head size normalization: reliability and validation against manual measurement of total intracranial volume. Neuroimage 2004;23:724-38

16. Gambardella A, Curcio A, Labate A, et al. Blocking out the real diagnosis. Lancet 2011;377:690

17. Abbott DF, Pell GS, Pardoe HR, et al. Selecting appropriate voxelbased methods for neuroimaging studies. Neuroimage 2012;59: 885-86 